\title{
Quantum Molecular Dynamics Approach to the Nuclear Matter Below the Saturation Density
}

\author{
Toshiki Maruyama ${ }^{1}$, Koji Niita ${ }^{1,2}$, Kazuhiro Oyamatsu ${ }^{3}$ Tomoyuki Maruyama $^{1}$, \\ Satoshi Chiba ${ }^{1}$, and Akira Iwamoto ${ }^{1}$, \\ 1 Advanced Science Research Center, \\ Japan Atomic Energy Research Institute, \\ Tokai, Ibaraki, 319-11 Japan \\ ${ }^{2}$ Research Organization for Information Science and Technology, \\ Tokai, Ibaraki, 319-11 Japan \\ ${ }^{3}$ Department of Energy Engineering and Science, Nagoya University \\ Furo-cho, Chikusa-ku, Nagoya 464-01, Japan
}

(April 4, 2018)

\begin{abstract}
Quantum molecular dynamics is applied to study the ground state properties of nuclear matter at subsaturation densities. Clustering effects are observed as to soften the equation of state at these densities. The structure of nuclear matter at subsaturation density shows some exotic shapes with variation of the density.
\end{abstract}

61.43.Bn,31.15.Qg,21.65.+f,26.20.+c,97.60.Jd,97.60.Bw,05.70.Fh 


\section{INTRODUCTION}

One of the main interests of heavy-ion physics and astrophysics is the property of nuclear matter in extreme conditions. Its high-density behavior is important for the scenario of supernova explosions, the evolution of neutron stars, the reaction process of high-energy heavy-ion collisions, quark-gluon plasma and so on. Properties of the nuclear matter below saturation density, on the other hand, are essential in describing the multi-fragmentation in the heavy-ion collisions, the collapsing stages in supernova explosions and the structure of neutron star crusts. Here the saturation density is the density of the energy-minimum state of the nuclear matter at a fixed proton ratio. For symmetric nuclear matter, the saturation density is the normal nuclear density $\rho_{0}=0.165 \mathrm{fm}^{-3}$. Since the matter is unstable below the saturation density, an inhomogeneous state is expected to appear below the saturation density.

Besides, supernova matter (SNM) and neutron star matter (NSM) are also interesting from the viewpoint of the nuclear shape. At low densities, nuclei in these matters are expected to be crystalized so as to minimize the long range Coulomb energies. They melt into uniform matter at a certain density close to the saturation density. Then what happens in between? About a decade ago, three groups [1] [3] suggested that nuclei have exotic structures in the SNM and/or NSM. They showed that the stable nuclear shape changes from sphere to cylinder, slab, cylindrical hole, and to spherical hole with increase of the matter density. The favorable nuclear shape is determined by a balance between the surface and Coulomb energies. In the liquid drop model, a simple geometrical argument demonstrates that the favorable shape changes as a function of the volume fraction of the nucleus in the cell, independently of specific nuclear interactions [2, [4]. ¿From recent studies in the liquid drop model [5] and the Thomas Fermi calculations [6, [7], the non-spherical shapes of nuclei are expected in a density range at about half the saturation density although the range depends on the choice of nuclear interaction. Furthermore these shapes are expected to survive even if the shell effects are taken into account [8]. 
These exotic nuclear shapes may cause substantial astrophysical consequences. In SNM, neutrino absorption by nuclei may modify leptonic energy of the matter and lead to a significant change in the strength of supernova explosions [5]. In neutron stars, the exotic nuclei may also affect the pinning of superfluid neutron vortices to nuclei in inner crusts of neutron stars, which are considered to be the initial step of neutron star glitches. Recently, Mochizuki and Izuyama [9] demonstrated that the exotic nuclear shapes actually play an essential role in trapping vortices in a microscopic mechanism of the glitches.

The spatial fluctuation is important in describing properties of SNM and NSM because geometrical distributions of nucleons may affect neutrino reaction rate in SNM and interactions with vortices in NSM. However, there have been only two works [3, 10] which allow arbitrary nuclear shapes in their models. These two works [3, 10] use the Thomas Fermi approximation and treat SNM while none has been done for NSM. Williams and Koonin [3] have investigated the structure of neutron-proton symmetric nuclear matter with the proton ratio $Z / A=0.5$, while the authors of Ref. [10] studied the matter with $Z / A=0.285$ at the total entropy per baryon $s=1$. Unfortunately, some spatial fluctuation due to a possible cluster correlation or non uniform distribution is neglected since their calculations with the Thomas-Fermi approximation are based on a one-body treatment of the matter.

In the field of nuclear reaction study, molecular dynamics has become one of the most powerful approach to simulate the fragmentation. Advantages of molecular dynamics for the investigation of heavy-ion reactions are that no reaction mechanism is required to assume and that the fluctuation of the system is automatically included.

Within various kinds of molecular dynamics, the quantum molecular dynamics (QMD) [11 16] approach has been proposed to study high energy heavy-ion collisions. QMD has been also used for the analysis of fusion reaction, nucleon-induced reaction, fragmentation in collisions between heavy systems, and so on.

In this paper, we apply the QMD method to the investigation of the equation of state (EOS) and the structure of nuclear matter at subsaturation densities. A similar calculation using QMD is done in Ref. [13] aiming to see the EOS of the nuclear matter. It was 
reported that the clustering in the matter significantly softens the EOS below saturation density. They have used, however, only 254 nucleons in a cell to simulate finite-temperature nuclear matter. This number seems insufficient to investigate the nuclear matter properties. We then perform the QMD calculation by using much more particles in a cell, and see the EOS of nuclear matter at zero-temperature.

In the present QMD, we introduce the Pauli potential and the momentum-dependent interaction in order to simulate the Fermionic feature in a phenomenological way and the energy-dependence of the optical potential. The binding energies of finite nuclei and the saturation properties of the nuclear matter are well adjusted to the empirical values. As a result of this new version of the QMD, we can approximately describe the properties of neutron-rich isotopes such as the density profile of ${ }^{11} \mathrm{Li}$ and the effects of surface neutrons in the low-energy collisions [17]. With these ingredients of the QMD and the periodic boundary condition, we investigate the ground state properties of the nuclear matter at subsaturation densities.

In Sect. II we describe our model based on the QMD. In Sect. III, the EOS and the structure of nuclear matter is discussed. Finally, summary is given in Sect. $\mathbb{}$.

\section{QMD SIMULATION FOR NUCLEAR MATTER}

The present QMD code is an improved version of our previous one [16]. In the previous works 16,18, where we have employed very simple effective interactions in the QMD, we have nicely reproduced several observables in the nucleon-induced reactions at energy region of about $100 \mathrm{MeV} \sim 5 \mathrm{GeV}$. Several improvements of this QMD are done to enable the calculations for the ground state of the nuclear matter for wide range of density and proton ratio. First we have introduced a phenomenological potential, namely Pauli potential to describe the Fermionic feature of nucleons. By this Pauli potential, we can uniquely determine the ground state of the finite nuclei and the nuclear matter by seeking the energy

minimum state. Second we have introduced momentum-dependent interaction, which is also 
an important feature of the Fermion system with finite range interaction. In the following, we explain the detail of the present QMD and how it describes the nuclear matter.

\section{A. The total wave-function and the equation of motion}

In QMD, each nucleon state is represented by a Gaussian wave-function of width $L$,

$$
\phi_{i}(\mathbf{r})=\frac{1}{(2 \pi L)^{3 / 4}} \exp \left[-\frac{\left(\mathbf{r}-\mathbf{R}_{i}\right)^{2}}{4 L}+\frac{i}{\hbar} \mathbf{r} \cdot \mathbf{P}_{i}\right]
$$

where $\mathbf{R}_{i}$ and $\mathbf{P}_{i}$ are the centers of position and momentum of $i$-th nucleon, respectively. The total wave-function is assumed to be a direct product of these wave-functions. Thus the one-body distribution function is obtained by the Wigner transform of the wave-function,

$$
\begin{gathered}
f(\mathbf{r}, \mathbf{p})=\sum_{i} f_{i}(\mathbf{r}, \mathbf{p}) \\
f_{i}(\mathbf{r}, \mathbf{p})=8 \cdot \exp \left[-\frac{\left(\mathbf{r}-\mathbf{R}_{i}\right)^{2}}{2 L}-\frac{2 L\left(\mathbf{p}-\mathbf{P}_{i}\right)^{2}}{\hbar^{2}}\right] .
\end{gathered}
$$

The equation of motion of $\mathbf{R}_{i}$ and $\mathbf{P}_{i}$ is given by the Newtonian equation

$$
\dot{\mathbf{R}}_{i}=\frac{\partial H}{\partial \mathbf{P}_{i}}, \quad \dot{\mathbf{P}}_{i}=-\frac{\partial H}{\partial \mathbf{R}_{i}}
$$

and the stochastic N-N collision term. Hamiltonian $H$ consists of the kinetic energy and the energy of the two-body effective interactions.

\section{B. Effective interactions}

The Hamiltonian is separated into several parts as follows,

$$
H=T+V_{\text {Pauli }}+V_{\text {local }}+V_{\mathrm{MD}},
$$

where $T, V_{\text {Pauli }}, V_{\text {local }}$ and $V_{\mathrm{MD}}$ are the kinetic energy, the Pauli potential, the local (momentum-independent) potential and the momentum-dependent potential parts, respectively. 
The Pauli potential [14, 19 21] is introduced for the sake of simulating Fermionic properties in a semiclassical way. This phenomenological potential prohibits nucleons of the same spin $\sigma$ and isospin $\tau$ from coming close to each other in the phase space. Here we employ the Gaussian form of the Pauli potential [14] as

$$
V_{\text {Pauli }}=\frac{1}{2} C_{\mathrm{P}}\left(\frac{\hbar}{q_{0} p_{0}}\right)^{3} \sum_{i, j(\neq i)} \exp \left[-\frac{\left(\mathbf{R}_{i}-\mathbf{R}_{j}\right)^{2}}{2 q_{0}^{2}}-\frac{\left(\mathbf{P}_{i}-\mathbf{P}_{j}\right)^{2}}{2 p_{0}^{2}}\right] \delta_{\tau_{i} \tau_{j}} \delta_{\sigma_{i} \sigma_{j}}
$$

In the local potential part we adopt the Skyrme type with the Coulomb and the symmetry terms as explained in Eq. (5) of Ref. 16,

$$
\begin{aligned}
V_{\text {local }}= & \frac{\alpha}{2 \rho_{0}} \sum_{i}<\rho_{i}>+\frac{\beta}{(1+\tau) \rho_{0}^{\tau}} \sum_{i}<\rho_{i}>^{\tau} \\
& +\frac{e^{2}}{2} \sum_{i, j(\neq i)} c_{i} c_{j} \iint d^{3} r_{i} d^{3} r_{j} \frac{1}{\left|\mathbf{r}_{i}-\mathbf{r}_{j}\right|} \rho_{i}\left(\mathbf{r}_{i}\right) \rho_{j}\left(\mathbf{r}_{j}\right) \\
& +\frac{C_{\mathrm{s}}}{2 \rho_{0}} \sum_{i, j(\neq i)}\left(1-2\left|c_{i}-c_{j}\right|\right) \rho_{i j}
\end{aligned}
$$

In the above equation, $c_{i}$ is 1 for protons and 0 for neutrons, while $\left\langle\rho_{i}>\right.$ is an overlap of density with other nucleons defined as

$$
\begin{aligned}
<\rho_{i}> & \equiv \sum_{j(\neq i)} \rho_{i j} \equiv \sum_{j(\neq i)} \int d^{3} r \rho_{i}(\mathbf{r}) \rho_{j}(\mathbf{r}) \\
& =\sum_{j(\neq i)}(4 \pi L)^{-3 / 2} \exp \left[-\left(\mathbf{R}_{i}-\mathbf{R}_{j}\right)^{2} / 4 L\right]
\end{aligned}
$$

The momentum-dependent term is introduced as a Fock term of the Yukawa-type interaction. We divide this interaction into two ranges so as to fit the effective mass and the energy dependence of the real part of the optical potential, as

$$
\begin{aligned}
V_{\mathrm{MD}} & =V_{\mathrm{MD}}^{(1)}+V_{\mathrm{MD}}^{(2)} \\
& =\frac{C_{\mathrm{ex}}^{(1)}}{2 \rho_{0}} \sum_{i, j(\neq i)} \frac{1}{1+\left[\frac{\mathbf{P}_{i}-\mathbf{P}_{j}}{\mu_{1}}\right]^{2}} \rho_{i j}+\frac{C_{\mathrm{ex}}^{(2)}}{2 \rho_{0}} \sum_{i, j(\neq i)} \frac{1}{1+\left[\frac{\mathbf{P}_{i}-\mathbf{P}_{j}}{\mu_{2}}\right]^{2}} \rho_{i j} .
\end{aligned}
$$

The parametrization of the constants included in the above effective interactions will be discussed in Sec. IIE. 


\section{Energy minimum state}

With inclusion of the Pauli potential, we can define the ground state as an energyminimum state of the system. To get the energy-minimum configuration, we use the following damping equation of motion,

$$
\dot{\mathbf{R}}_{i}=\frac{\partial H}{\partial \mathbf{P}_{i}}+\mu_{\mathbf{R}} \frac{\partial H}{\partial \mathbf{R}_{i}}, \quad \dot{\mathbf{P}}_{i}=-\frac{\partial H}{\partial \mathbf{R}_{i}}+\mu_{\mathbf{P}} \frac{\partial H}{\partial \mathbf{P}_{i}},
$$

where $\mu_{\mathbf{R}}$ and $\mu_{\mathbf{P}}$ are the damping coefficients with negative values when we need to cool the system.

We first distribute the particles randomly in the phase space and cool down the system according to the damping equation of motion until the energy reaches the minimum value. Sometimes the system stops at the local minimum during the cooling. We thus try again and again this cooling procedure with a different initial state and seek the real energy minimum state.

For finite nucleus and infinite system above saturation density, this procedure works rather well. For infinite system at subsaturation densities, however, there are many local minimum states around the real ground state, which differ from the ground state in the detail of the surface configuration of the clusters. Since the energy difference from the ground state is the order of $10 \mathrm{keV} /$ nucleon in this case, we accept these states as ground states and neglect the small differences of the configuration.

\section{Periodic boundary condition}

In order to simulate infinite nuclear matter with finite number of particles, we use a cubic cell with periodic boundary condition. The size of the cell is determined from the average density and the particle number. The periodic boundary condition is introduced as follows: As shown in Fig. 1, we prepare $26\left(=3^{3}-1\right)$ surrounding cells, where the particle distribution reflects the distribution of the central cell exactly. The particles in the central cell move according to the interaction with all particles in the same cell and in the surrounding cells 
as well. The particles in the surrounding cells obey exactly the same motions as those in the central cell. Thus the Hamiltonian per cell is written as

$$
H=\sum_{i=1, \cdots, N}\left[T_{i}+\sum_{\substack{\text { cell }=0, \cdots, 26 \\ j=1, \cdots, N}} H_{i j}^{(2)}\left(\mathbf{R}_{i}-\mathbf{R}_{j}+\mathbf{D}_{\text {cell }}, \mathbf{P}_{i}, \mathbf{P}_{j}\right)+\cdots\right]
$$

where $T_{i}$ is one-body part (kinetic energy), $H_{i j}^{(2)}$ is the two body part of the Hamiltonian and $\mathbf{D}_{\text {cell }}$ are the relative position of surrounding cells from the center. Note that the indices "cell" runs from 0 (the center cell) to 26 (surrounding cells) and $\mathbf{D}_{0}=0$.

\section{E. Parametrization of the constants}

We have twelve parameters in the effective interactions of the Hamiltonian Eq. (5), i.e., $C_{\mathrm{P}}, q_{0}, p_{0}, \alpha, \beta, \tau, C_{\mathrm{s}}, C_{\mathrm{ex}}^{(1)}, C_{\mathrm{ex}}^{(2)}, \mu_{1}, \mu_{2}$ and the Gaussian width $L$. We should parametrize these constants to reproduce properties of the ground states of the finite nuclei and saturation properties of the nuclear matter.

We first determine the parameters of Pauli potential $q_{0}, p_{0}$ and $C_{\mathrm{P}}$, apart from the other effective interactions, by fitting the kinetic energy of the exact Fermi gas at zero temperature and at various densities. For this, we define the free Fermi gas system as a ground state for the Hamiltonian including only the kinetic energy and the Pauli potential by making use of the damping equation of motion Eq. (10) and the periodic boundary condition with 1024 particles in a cell. In Fig. 2, we show the kinetic energies (the solid squares) and the total energies (the open squares) obtained by using a parameter set for the Pauli potential as

$$
C_{\mathrm{P}}=207 \mathrm{MeV}, \quad p_{0}=120 \mathrm{MeV} / c, \quad q_{0}=1.644 \mathrm{fm} .
$$

In the same figure we draw the exact energy of the Fermi gas by the solid line. Although there are some other parameter sets which can reproduce the exact energies of the Fermi gas in the same form, i.g. that used in Ref. [13], we choose the above parameter set to get good properties of the ground states of the finite nuclei with other effective interactions particularly in combination with the momentum-dependent interaction. 
Among remaining nine conditions, four are attributed to the momentum-dependent interaction as follows. We calculate the single particle potential of momentum $\mathbf{p}$ in ideal nuclear matter at the normal nuclear density, which leads to

$$
\begin{aligned}
U\left(\mathbf{p}, \rho_{0}\right) & =U_{\text {local }}+U_{\mathrm{MD}}(\mathbf{p}) \\
& =\alpha+\beta+\left(\frac{4}{3} \pi p_{\mathrm{F}}^{3}\right)^{-1} \int^{p_{\mathrm{F}}} d^{3} p^{\prime}\left[\frac{C_{\mathrm{ex}}^{(1)}}{1+\left[\frac{\mathbf{p}-\mathbf{p}^{\prime}}{\mu_{1}}\right]^{2}}+\frac{C_{\mathrm{ex}}^{(2)}}{1+\left[\frac{\mathbf{p}-\mathbf{p}^{\prime}}{\mu_{2}}\right]^{2}}\right] \\
& =\alpha+\beta+C_{\mathrm{ex}}^{(1)} g\left(x=\mu_{1} / p_{\mathrm{F}}, y=p / p_{\mathrm{F}}\right)+C_{\mathrm{ex}}^{(2)} g\left(x=\mu_{2} / p_{\mathrm{F}}, y=p / p_{\mathrm{F}}\right),
\end{aligned}
$$

with

$$
g(x, y)=\frac{3}{4} x^{3}\left[\frac{1+x^{2}-y^{2}}{2 x y} \ln \frac{(y+1)^{2}+x^{2}}{(y-1)^{2}+x^{2}}+\frac{2}{x}-2\left\{\arctan \frac{y+1}{x}-\arctan \frac{y-1}{x}\right\}\right] .
$$

We fit the energy-dependence of this potential to the experimental data. In Fig. 3, we plot the energy dependence of the real part of the optical potential (the open circles and squares) obtained from the experimental data of Hama et al. 22 for p-nucleus elastic scattering. ¿From this figure, we pick up three conditions, i.e. $U(0)=-80 \mathrm{MeV}, U(p)=0$ at $E_{\text {lab }}=200$ $\mathrm{MeV}$, and $U(p \rightarrow \infty)=\alpha+\beta=77 \mathrm{MeV}$. For another condition, we use the value of effective mass defined by

$$
\frac{1}{m^{*}}=\frac{1}{m}+\left(\frac{1}{p} \frac{\partial U_{\mathrm{MD}}}{\partial p}\right)_{p=p_{\mathrm{F}}}
$$

We take the value of $m^{*}=0.8 m$ at $\rho=\rho_{0}$.

Other three conditions are coming from the saturation condition, i.e. the energy per nucleon $E / A=-16 \mathrm{MeV}$ at $\rho=\rho_{0}\left(0.165 \mathrm{fm}^{-3}\right)$ and the value of incompressibility $K$.

The last two parameters are given by hand. One is the value of the coefficient of the symmetry term $C_{\mathrm{s}}$. We take $25 \mathrm{MeV}$ for $C_{\mathrm{s}}$. This value leads to the symmetry energy 34.6 $\mathrm{MeV}$ for the nuclear matter at the saturation density (see Sec. [IIB). The other is the width of the Gaussian wave packet $L$, which is a free parameter in QMD model. This value affects ground state properties of finite nuclei and infinite nuclear matter below saturation densities, while it does not change those of infinite nuclear matter above saturation densities. We then choose this value to give nice fitting the binding energies of finite nuclei. 
It should be noted here that we cannot determine these parameters from the above conditions in an analytical way, since the Fermi distribution is not exactly achieved by the Pauli potential and the additional potential energy included in the Pauli potential. In addition, the saturation properties of the nuclear matter should be realized in the simulated matter for the main purpose of this paper. We then simulate the nuclear matter by the QMD with the periodic boundary condition using 1024 particles in a cell. We search the energy minimum state by the damping equation of motion as discussed above and adjust the parameters. By this method, we have fixed three parameter sets according to the value of incompressibility $K$, which are shown in Table 1 . We have prepared three kinds of equation of state, namely Soft $(K=210 \mathrm{MeV})$, Medium $(K=280 \mathrm{MeV})$ and Hard $(K=380 \mathrm{MeV})$ EOS. These values of incompressibility $K$ are subtracted from the results of EOS (shown in Fig. 6) by fitting its curvature at the saturation point to the following parabolic form,

$$
E / A=\frac{K}{18 \rho_{0}^{2}}\left(\rho-\rho_{0}\right)^{2}-16 .
$$

This parabola is also shown in Fig. 6.

Here the single particle potential shown in Fig. 3 are also calculated by the simulated nuclear matter with Pauli potential and other effective interactions instead of ideal nuclear matter. The results are denoted by the crosses in Fig. [3 and well coincident with the results of ideal nuclear matter except for the low energy part, where the Pauli potential is effective. Though this result in Fig. 3 is obtained with a parameter set of Medium EOS, results with Soft and Hard EOS are same as Medium EOS within $2 \mathrm{MeV}$ for all energy region.

In Fig. 因, we plot the binding energies of the ground state of finite nuclei obtained by the damping equation of motion Eq. (10) with three parameter sets, i.e., Soft (the long dashed line), Medium (the dashed line) and Hard (the solid line) EOS. All of them reproduce well the global trend of the binding energies of various nuclei except for light nuclei from ${ }^{12} \mathrm{C}$ to ${ }^{20} \mathrm{Ne}$. It might be due to the specific structures of these light nuclei, which are not well described by the present QMD. 


\section{F. "Screened" Coulomb potential}

For the neutron star, the same number of electrons exist as proton, since the nuclear matter in the neutron star should be charge-neutral. Hence the energy of the system remains finite even if we calculate the Coulomb interaction of protons and electrons. However, Coulomb interaction has so long range that the Coulomb energy depends on the cell-size in our treatment of the infinite system by the periodic boundary condition with the surrounding neighbor cells. To avoid this cell-size dependence, we introduce a cutoff of the Coulomb interaction in the way of "screened" Coulomb potential. We use the following "screened" Coulomb interaction instead of the second term of Eq. (77) for the nuclear matter calculations,

$$
V_{\mathrm{C}}^{\mathrm{scr}}=\frac{e^{2}}{2} \sum_{i, j(\neq i)} c_{i} c_{j} \iint d^{3} r_{i} d^{3} r_{j} \frac{\exp \left[-\left|\mathbf{r}_{i}-\mathbf{r}_{j}\right| / r_{\mathrm{scr}}\right]}{\left|\mathbf{r}_{i}-\mathbf{r}_{j}\right|} \rho_{i}\left(\mathbf{r}_{i}\right) \rho_{j}\left(\mathbf{r}_{j}\right) \text {. }
$$

In this equation, $r_{\text {scr }}$ is the "screening" length, which we use $10 \mathrm{fm}$ in the present study. The physical screening of the Coulomb potential by the electron localization is, however, estimated to be much larger in the case of normal nuclear density [3]. Thus our "screening" should be considered as a technical approximation to avoid this cell-size dependence and to make the numerical calculation feasible. For this purpose, $r_{\text {scr }}$ should be smaller than the cell size. On the other hand, to keep the proper description of finite nuclei, it should be lager than the size of nuclei. By the "screened" Coulomb interaction with $r_{\text {scr }}=10 \mathrm{fm}$, however, the binding energies are slightly modified particularly in heavy nuclei. We compare the binding energies obtained by the "screened" Coulomb interaction with that of the normal one in Fig. 4. The dotted line is the result of the "screened" Coulomb interaction with Medium EOS. Though the binding energies of heavy nuclei increase, the binding energies

of finite nuclei still have the maximum around at $A \approx 100$. This feature is important to describe clustering of the matter at low densities. 


\section{EQUATION OF STATE AND THE STRUCTURE OF NUCLEAR MATTER}

In this section we study properties of nuclear matter at several conditions. It is desirable to use a cell large enough to include several periods of structure and to avoid the spurious effects of boundary condition on the structure of the matter. Though our calculation with typically 1024 particles in a cell is not fully satisfactory in this respect, we consider it is enough for semiqualitative discussions at the beginning of this study. Actually, the global quantities, i.g. the ground state energy of the system, is well saturated at this number of particles in a cell. In Fig. 5, we show the energy per nucleon of the infinite system as a function of the particle number in a cell for four average densities from $\rho=0.4 \rho_{0}$ to $2.2 \rho_{0}$. For all densities, the energy of the system have already approached the asymptotic value above 256 particles within $100 \mathrm{keV} /$ nucleon. In this study we then simulate the infinite system by the periodic boundary condition with 1024 or 2048 particles in a cell, and investigate ground state properties of the nuclear matter.

\section{A. Symmetric nuclear matter}

We first perform the calculations for symmetric $(Z / A=0.5)$ matter at zero temperature to simulate supernova matter (SNM) in the collapsing stage. Figure 6 shows the energy per nucleon as a function of the average density. The solid squares indicate the energy of "uniform" nuclear matter while open squares are the results of energy-minimum configurations.

The "uniform" matter energy is calculated as follows: First we distribute nucleons randomly and cool the system only with the Pauli potential. Pauli potential is repulsive and does not spoil the uniformity of the system. Then we impose the other effective interactions

and cool only in the momentum space fixing the positions of particles. The system turns out to be approximately uniform with this procedure. Note that the simulated "uniform" matter is not exactly the same as ideal nuclear matter since the latter is continuous and completely uniform. 
Both cases of uniform and energy-minimum configurations have almost the same energy per nucleon for the higher densities as is seen in this figure. Below saturation density $\rho_{0}$, the energy per nucleon of the energy-minimum configuration is lower than the uniform case. The deviation amounts to about $5 \mathrm{MeV}$. As we see in the following, this is due to the structure change of the matter from uniform to non uniform structure such as clusterized one.

This change of the structure is displayed in Fig. 7. Above $0.8 \rho_{0}$, the system is almost uniform and no specific structure is seen. Below $0.8 \rho_{0}$, however, there appear some voids between the matter. As the density gets lower, the voids develop and nuclei are surrounded by the voids. Below $0.2 \rho_{0}$ each nucleus is separate, while above that density nuclei are connected to form some transient structures. This change of the structure is basically the same as reported in the previous works [1] [3]. Furthermore, the transient shape of the matter like hole, slab and cylinder are partly seen in our calculation although the nuclear surface shape in QMD is somewhat complicated. It should be noted that the nuclear shapes do not show exact symmetry properties (sphere, cylinder or slab) assumed in the previous liquid-drop and Thomas-Fermi studies [1.2:4] [], where the assumption of the symmetries leads to clear changes of the nuclear shape. Our result suggests that during the transition from homogeneous to inhomogeneous matter, the nuclear shape may not have these simple symmetry properties. It is possible that this is due to the incomplete minimization of the energy because there are several local minimums around the real ground state and the energy difference due to the nuclear shape is extremely small. However, these shapes are not so strange in the transient SNM at the collapsing stage because the matter is not in perfect equilibrium.

The change of the shape obtained in the QMD calculation may affect the neutrino reaction rate in SNM. It has been pointed out [1] 3] that the nuclear distributions are essentially determined by a delicate balance (of the order of $1 \mathrm{keV} /$ nucleon) between the surface and Coulomb energies. In the present treatment, however, we neglect such a tiny energy difference between the nuclear shapes around the ground state. We then need further improvement of our treatment to investigate the affect of the shape on the neutrino reaction rate in SNM. 
Nevertheless, our results provide the EOS of the symmetric matter with sufficient accuracy as well as global nuclear structure in the matter.

\section{B. Asymmetric nuclear matter}

In the neutron star matter (NSM), the beta-equilibrium is achieved and the proton ratio is given by the energy-minimum condition. The left panel of Fig. 8 is the energy per nucleon of nuclear matter with several proton ratios. The electron kinetic energy is not included in this figure, though the Coulomb interaction of protons and uniform electron background is included. The energy per nucleon at $\rho=\rho_{0}$ is fitted by

$$
E / A \approx-16.2+34.6 \frac{(Z-N)^{2}}{A^{2}}[\mathrm{MeV}]
$$

In other words, the symmetry energy at normal density is $34.6 \mathrm{MeV}$ in our calculation. The symmetry energies at $\rho=0.6 \rho_{0}$ and $0.2 \rho_{0}$ obtained in the same way are 23.0 and $18.9 \mathrm{MeV}$, respectively.

Including the electron kinetic energy at zero temperature, we get the total energy of NSM as a function of the proton ratio shown in the right panel of Fig. 8. It can be seen from this figure that the proton ratio that gives the energy-minimum of the system is 0.032 $\left(\rho=0.2 \rho_{0}\right), 0.043\left(\rho=0.6 \rho_{0}\right)$ and $0.072\left(\rho=1.0 \rho_{0}\right)$.

The structure of asymmetric nuclear matter at low density $\left(0.1 \rho_{0}\right)$ is shown in Fig. 9 . Even though the proton ratio is small, nucleons form the cluster structure at low density. If the proton ratio is very small, some neutrons can not stay inside the cluster but overflow into the space; clusters are floating in the neutron sea. When the proton ratio increases, free neutrons are absorbed into the clusters.

The departure from the spherical symmetry of the nuclear (cluster) shape is also seen as in the symmetric matter. This may cause some consequences in the standard scenario of pulsar glitches. In the scenario, vortices in the superfluid neutron sea are supposed to be pinned to clusters (nuclei) and accumulated in the inner crusts because neutrons are normal 
in nuclei. The strength of the pinning, which is important in the scenario, depends on the geometry of nuclei as well as on the superfluid energy gap.

Finally, we have to recognize that the simulation of infinite system still needs much lager number of particles. At least a cell must include several periods of structure for distinct conclusions. If only one or two units of the structure is included in a cell, the size of the unit structure is the same or half size of the given cell size. The lattice is also limited to be cubic. In this case, the results are dependent on the boundary condition which is artificially imposed. However, we emphasize that the QMD framework can be used also for the NSM and the symmetric matter as has been used for the reaction studies. Together with some refinements of the surface energy, the present method will be able to describe from stable and unstable nuclei to homogeneous and inhomogeneous nuclear matter. The problem of the computational time in this study is expected to be solved soon.

\section{SUMMARY}

We have proposed the QMD approach for the description of nuclear matter in wide range of density and proton ratio. We can well reproduce the finite nuclear properties for various mass range by inclusion of the Pauli potential and the momentum-dependent interaction. We have investigated the EOS of nuclear matter by simulating the infinite system with our QMD. Below the saturation density, clustering of the system was observed, which softens the EOS by lowering the energy per nucleon up to about $5 \mathrm{MeV}$.

We have shown the structure of the nuclear matter at subsaturation density. The transient shape of the symmetric nuclear matter, such as hole, slab, cylinder and sphere, predicted in previous works with analytic model and Thomas-Fermi calculations [1 [3], are partially seen in our calculation. However the structure of nuclear matter at subsaturation density appears rather vaguely in our case. This result suggests that during the transition from homogeneous to inhomogeneous matter, the nuclear shape may not have these simple symmetry properties. We need, however, further investigation increasing the particle 
number to get quantitative conclusion.

For asymmetric nuclear matter we have obtained the proton ratios $Z / A=0.032(\rho=$ $\left.0.2 \rho_{0}\right), 0.043\left(\rho=0.6 \rho_{0}\right)$ and $0.072\left(\rho=1.0 \rho_{0}\right)$, which give the energy-minimum of the system for the fixed average densities. At considerably low proton ratio, we have observed a neutron sea in which the normal nuclei are floating around.

In this paper we have presented our first results on the infinite nuclear matter by the use of molecular dynamics method. Though it is still necessary to enlarge the particle number, our results agree quantitatively to previous studies which include much more assumptions and restrictions in the models.

Our model contains further possibility for the simulation of dynamical evolution of infinite nuclear matter such as supernova explosion, the glitch of the neutron star and the initial stage of the universe. Intensive and systematic study of the nuclear matter with present model will be important since it contains less assumption than the foregoing models as to the structure of the matter. 


\section{REFERENCES}

[1] D. G. Ravenhall, C. J. Pethick and J. R. Wilson, Phys. Rev. Lett. 27 (1983) 2066.

[2] M. Hashimoto, H. Seki and M. Yamada, Prog. Theor. Phys. 71 (1984) 320.

[3] R. D. Williams and S. E. Koonin, Nucl. Phys. A435 (1985) 844.

[4] K. Oyamatsu, M. Hashimoto and M. Yamada, Prog. Theor. Phys. 71 (1984) 320.

[5] C. P. Lorentz, D. G. Ravenhall and C. J. Pethick, Phys. Rev. Lett. 25 (1993) 379.

[6] K. Oyamatsu, Nucl. Phys. A561 (1993) 431.

[7] K. Sumiyoshi, K. Oyamatsu and H. Toki, Nucl. Phys. A595 (1995) 327.

[8] K. Oyamatsu and M. Yamada, Nucl. Phys. A578 (1994) 181.

[9] Y. Mochizuki and T. Izuyama, Astrophys. J. 440 (1995) 263.

[10] M. Lassaut, H. Flocard, P. Bonche, P. H. Heenen and E. Surand Astron. Astrophys. 183 (1987) L3.

[11] J. Aichelin and H. Stöcker, Phys. Lett. B 176 (1986) 14.

[12] J. Aichelin, Phys. Rep. 202 (1991) 233; and references therein.

[13] G. Peilert, J. Randrup, H. Stoc̈ker and W. Greiner, Phys. Lett. B 260 (1991) 271.

[14] G. Peilert, J. Konopka, H. Stöker, W. Greiner, M. Blann and M. G. Mustafa, Phys. Rev. C 46 (1992) 1457.

[15] T. Maruyama, A. Ono, A. Ohnishi and H. Horiuchi, Prog. Theor. Phys. 87 (1992) 1367.

[16] K. Niita, S. Chiba, T. Maruyama, T. Maruyama, H. Takada, T. Fukahori, Y. Nakahara and A. Iwamoto, Phys. Rev. C 52 (1995) 2620.

[17] K. Niita et al., in preparation.

[18] M. B. Chadwick, S. Chiba, K. Niita, T. Maruyama and A. Iwamoto, Phys. Rev. C 52, 
2800 (1995); S. Chiba, M. B. Chadwick, K. Niita, T. Maruyama and A. Iwamoto, Phys. Rev. C 53, 1824 (1996); S. Chiba, O. Iwamoto, T. Fukahori, K. Niita, T. Maruyama, T. Maruyama and A. Iwamoto, Phys. Rev. C 54, 285 (1996).

[19] D. H. Boal and J. N. Glosli, Phys. Rev. C 38 (1988) 1870.

[20] A. Ohnishi, T. Maruyama and H. Horiuchi, Prog. Theor. Phys. 87 (1992) 417.

[21] T. Maruyama, K. Niita, and A. Iwamoto, Phys. Rev. C 53 (1996) 297.

[22] S. Hama, B. C. Clark, E. D. Cooper, H. S. Scherif and R. L. Mercer, Phys. Rev. C 41 (1990) 2737. 


\section{TABLES}

TABLE I. Effective interaction parameter set

\begin{tabular}{cccc}
\hline \hline & Soft $(K=210 \mathrm{MeV})$ & Medium $(K=280 \mathrm{MeV})$ & Hard $(K=380 \mathrm{MeV})$ \\
\hline$\alpha(\mathrm{MeV})$ & -223.56 & -92.86 & -21.21 \\
$\beta(\mathrm{MeV})$ & 298.78 & 169.28 & 97.93 \\
$\tau$ & 1.16667 & 1.33333 & 1.66667 \\
$C_{\mathrm{s}}(\mathrm{MeV})$ & 25.0 & 25.0 & 25.0 \\
$C_{\mathrm{ex}}^{(1)}(\mathrm{MeV})$ & -258.54 & -258.54 & -258.54 \\
$C_{\mathrm{ex}}^{(2)}(\mathrm{MeV})$ & 375.6 & 375.6 & 375.6 \\
$\mu_{1}(\mathrm{MeV})$ & 2.35 & 2.35 & 2.35 \\
$\mu_{2}(\mathrm{MeV})$ & 0.4 & 0.4 & 0.4 \\
$L\left(\mathrm{fm}^{2}\right)$ & 2.1 & 2.1 & 2.05 \\
\hline \hline
\end{tabular}




\section{FIGURES}

FIG. 1. An illustrative explanation of cell configuration. 26 surrounding cells (only 8 cells are displayed in this figure) have exactly the same distribution of particles as the central cell. The relative position vector of each surrounding cell from the central cell 0 is $\mathbf{D}_{\text {cell }}$ and $\mathbf{D}_{0}=0$.

FIG. 2. Energy per particle of free Fermi gas. The solid line shows the exact value. The cases of the molecular dynamics calculation with only Pauli potential are shown by the solid squares (kinetic energy) and the open squares (total energy).

FIG. 3. The energy dependence of the real part of the optical potential. The open circles and squares indicate the results obtained from the experimental data of Hama et al. 22 for p-nucleus elastic scattering. The solid line denotes the single particle potential calculated by Eq. (13) in ideal nuclear matter with the parameter set of Medium EOS. The short and long dashed lines

show $\alpha+\beta+C_{\mathrm{ex}}^{(1)} g(x, y)$ and $C_{\mathrm{ex}}^{(2)} g(x, y)$ of Eq. (13), respectively. The single particle potential calculated by the simulated nuclear matter with Pauli potential is shown by the crosses.

FIG. 4. Binding energies of finite system obtained by the damping equation of motion with three parameter sets, i.e., Soft (the long dashed line), Medium (the dashed line) and Hard (the solid line) EOS. The solid squares denote experimental data. The dotted line indicates the binding energy per nucleon obtained by using the "screened" Coulomb interaction in the case of Medium EOS.

FIG. 5. Particle number dependence of the energy per nucleon of the infinite system for four average densities from $\rho=0.4 \rho_{0}$ to $2.2 \rho_{0}$. 
FIG. 6. The energy per nucleon of symmetric nuclear matter $(Z / A=0.5)$ at zero temperature as a function of the average densities. ¿From the left, the open squares are the results with Soft $(K=210 \mathrm{MeV})$, Medium $(K=280 \mathrm{MeV})$ and Hard EOS $(K=380 \mathrm{MeV})$ obtained by the damping equation of motion searching the energy-minimum configuration in the full phase-space. The solid squares indicate results obtained from the spatially uniform distribution. The kinetic energy of the electron is not included. We use 1024 particles in a cell for all cases.

FIG. 7. The structure of symmetric nuclear matter. ¿From the upper left, the average density is $\rho=1.0 \rho_{0}, 0.8 \rho_{0}, 0.6 \rho_{0}, 0.4 \rho_{0}, 0.2 \rho_{0}$ and $0.1 \rho_{0}$. The white circles denote neutrons and red circles are protons. Nuclear potential of Medium EOS is used. We use 2048 particles in a cell for these cases and the size of a cell is indicated in the figure.

FIG. 8. The left panel: the energy per nucleon of nuclear matter as a function of the proton ratio $Z / A$ for the three fixed average densities $\rho=0.2 \rho_{0}$ (the solid triangles), $0.6 \rho_{0}$ (the solid squares), and $1.0 \rho_{0}$ (the solid circles). The right panel: same as the left panel but with the kinetic energy of the electrons.

FIG. 9. The structure of asymmetric nuclear matter. ¿From the upper left, the proton ratio $Z / A$ is $0.098,0.195,0.293$ and 0.391 , while the average density is $0.1 \rho_{0}$ for all panels. The white circles denote neutrons and red circles are protons. Nuclear potential of Medium EOS is used. We use 1024 particles in a cell for these cases and the size of a cell is indicated in the figure. 


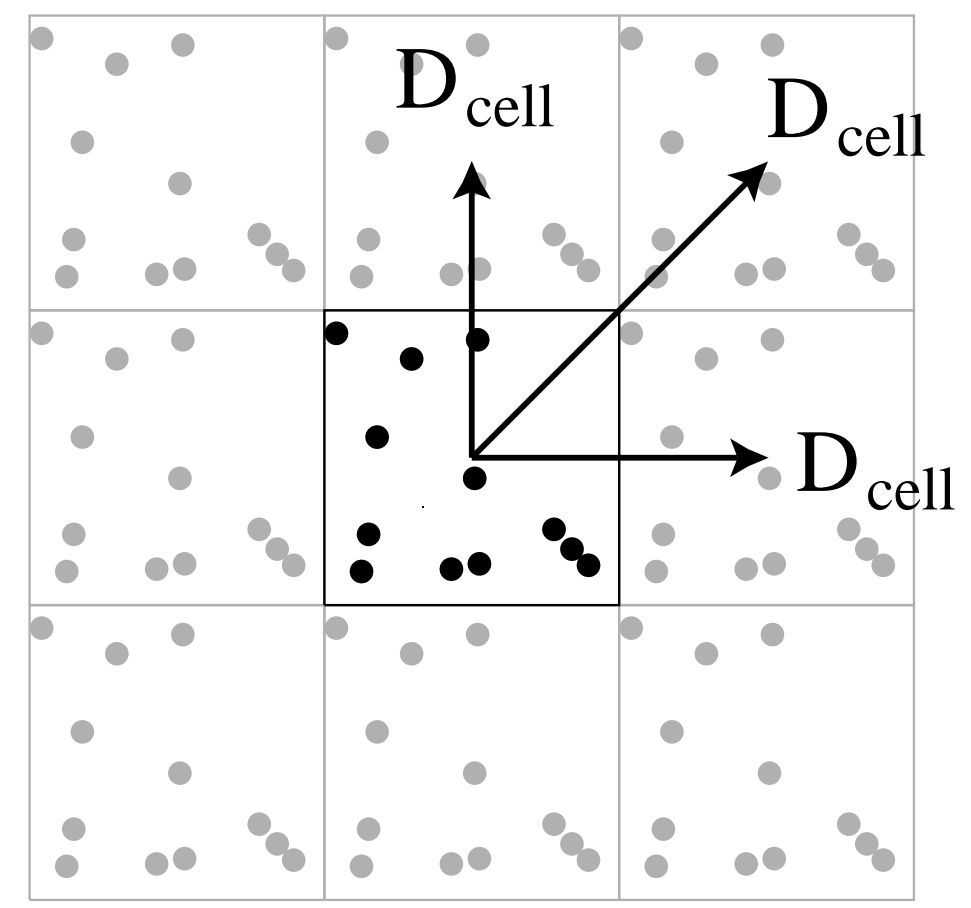




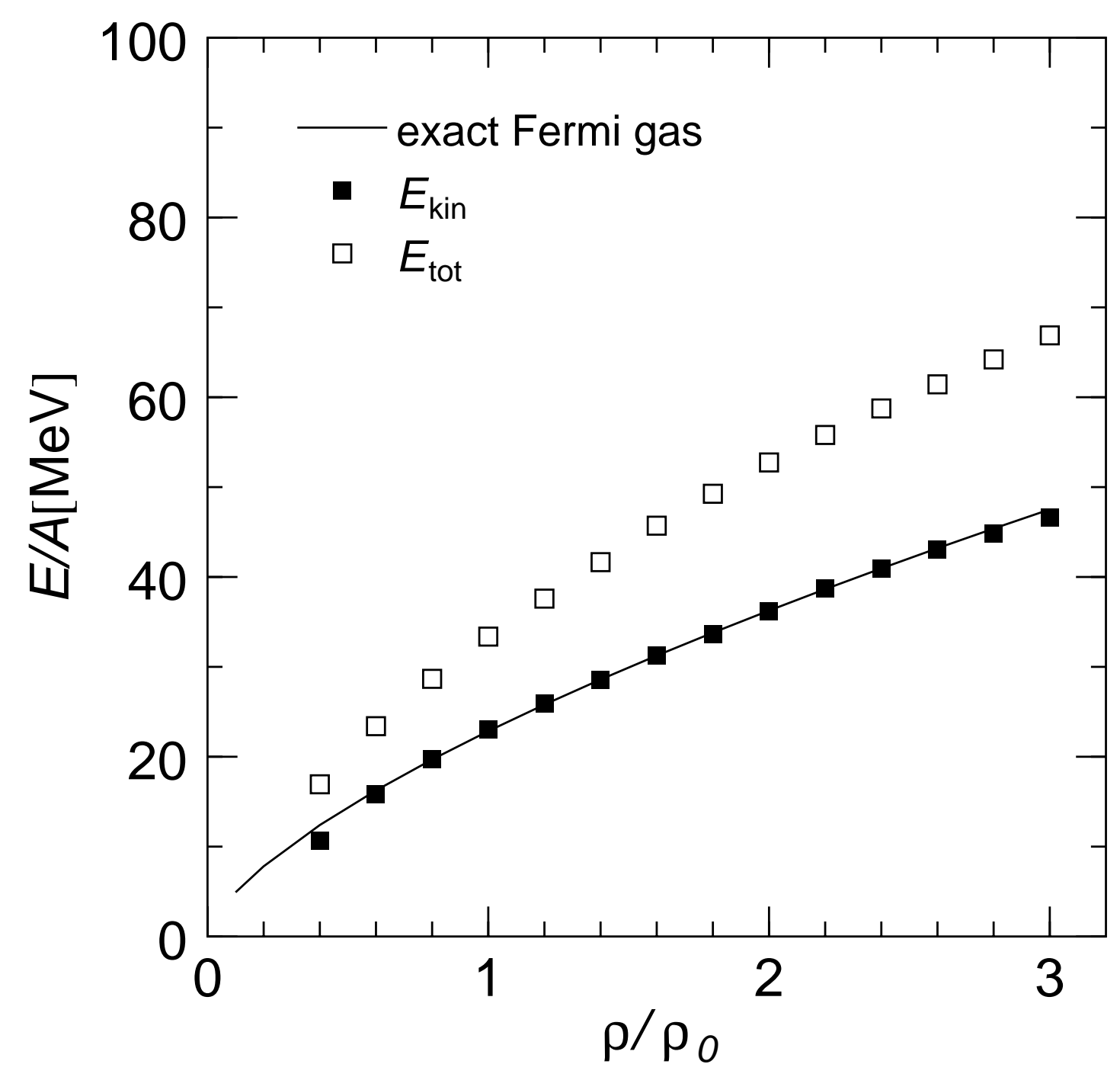




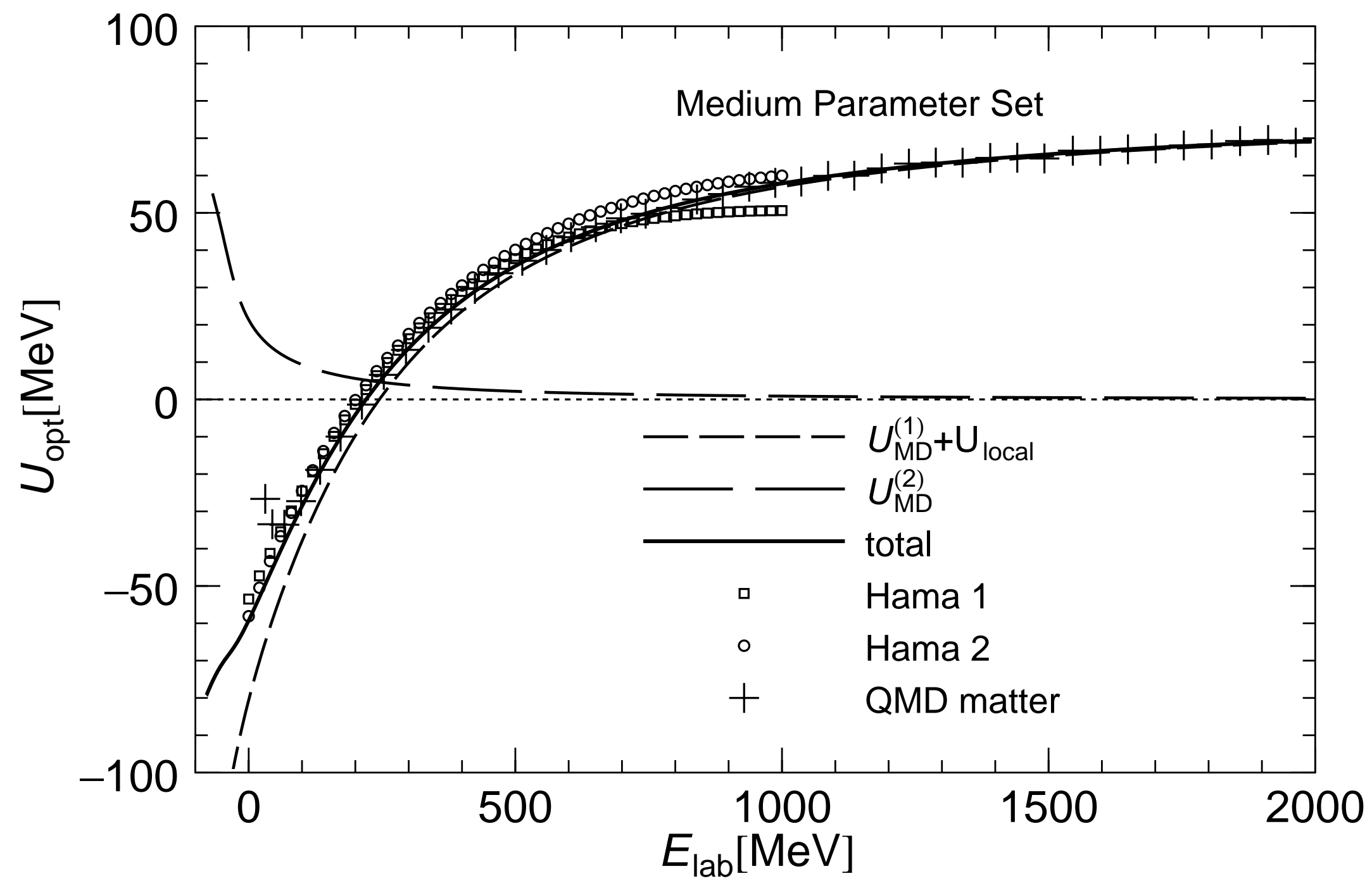




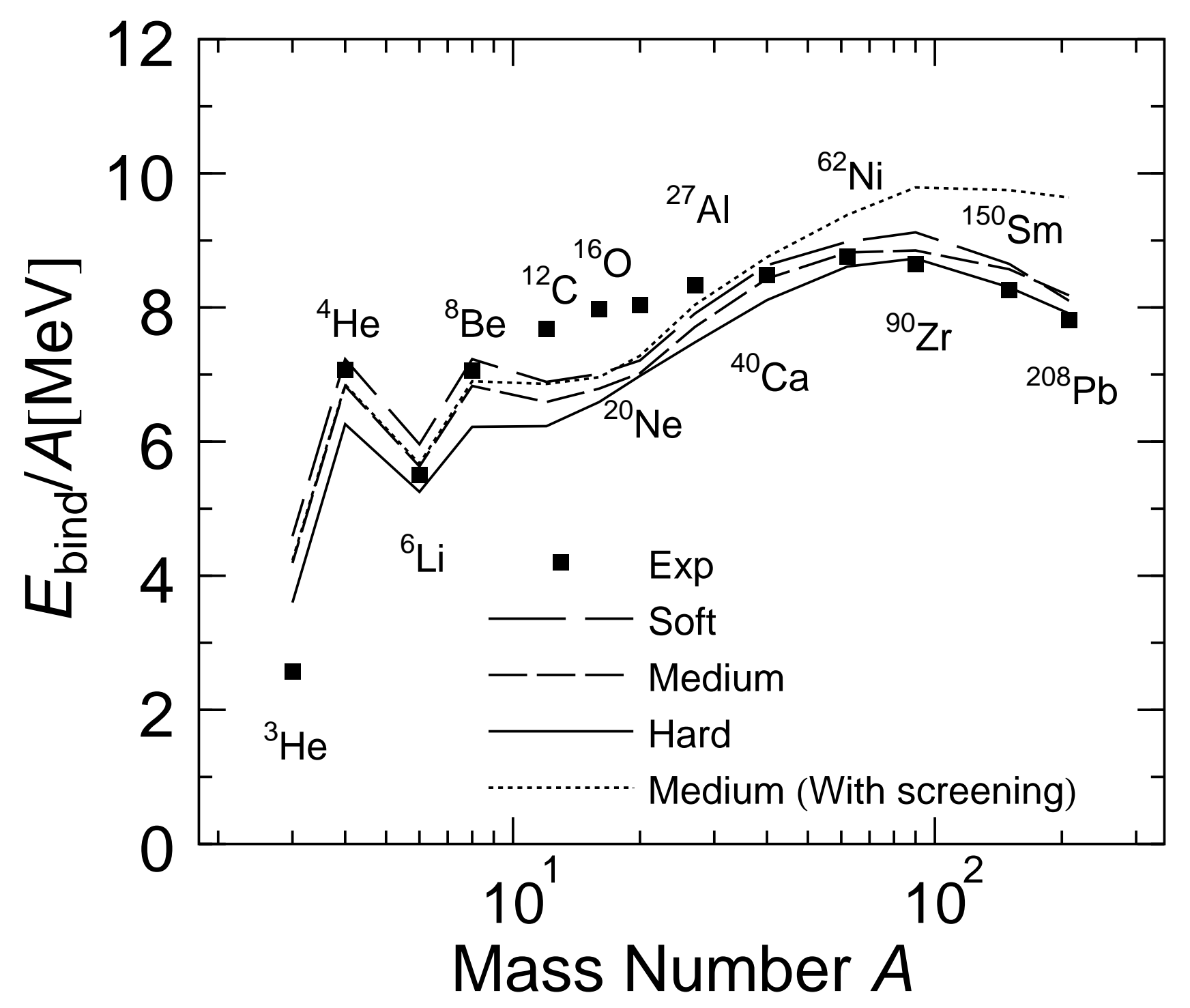




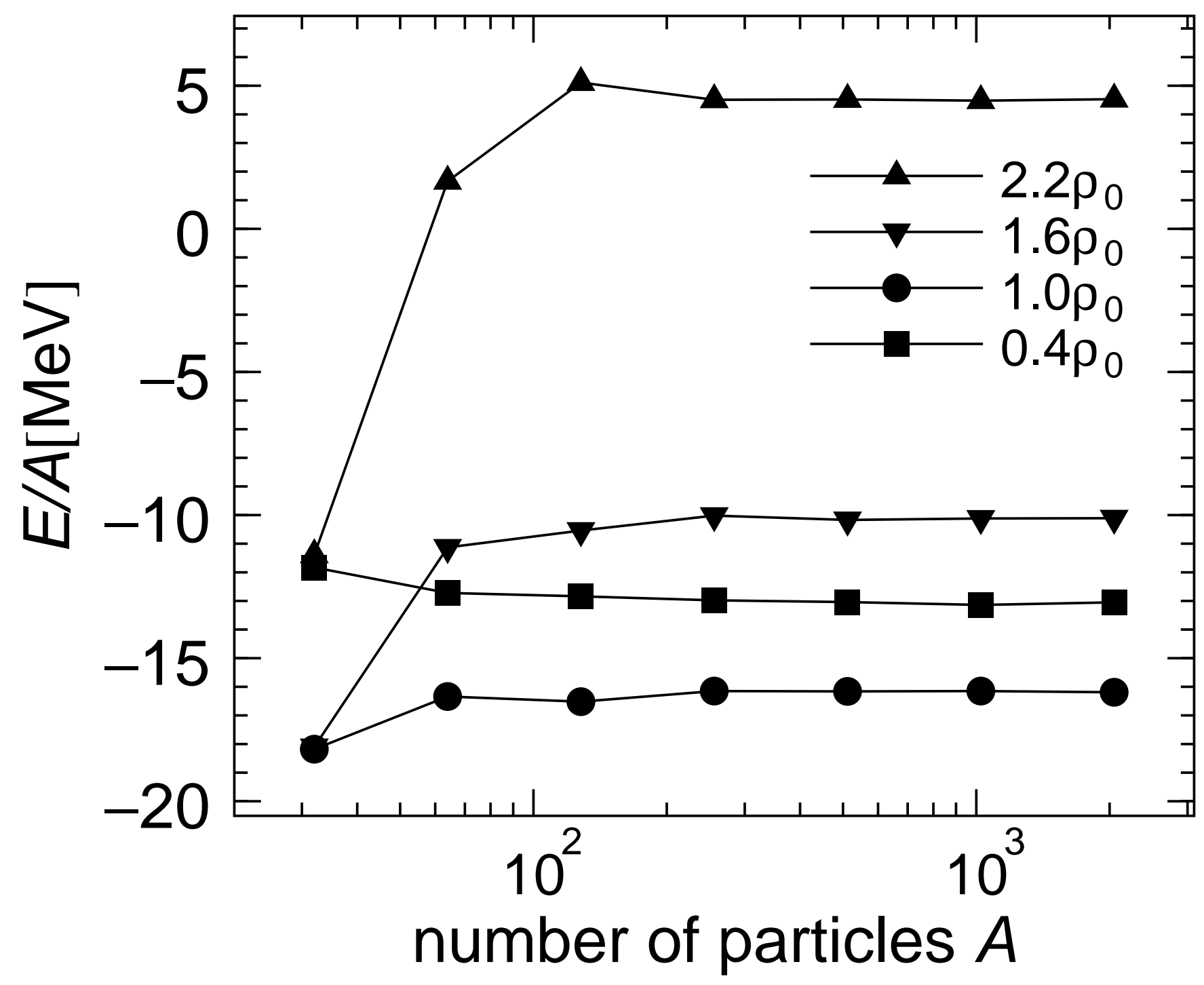




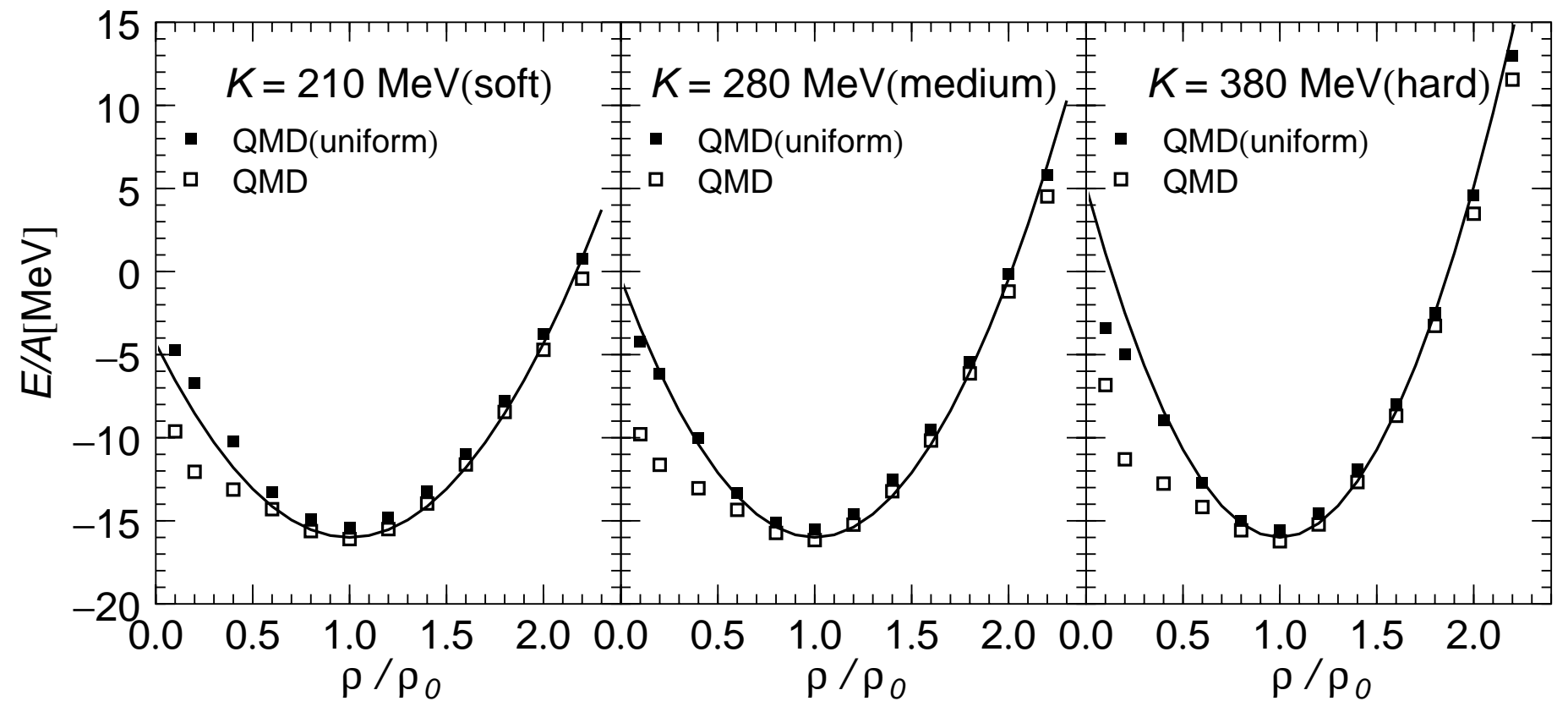



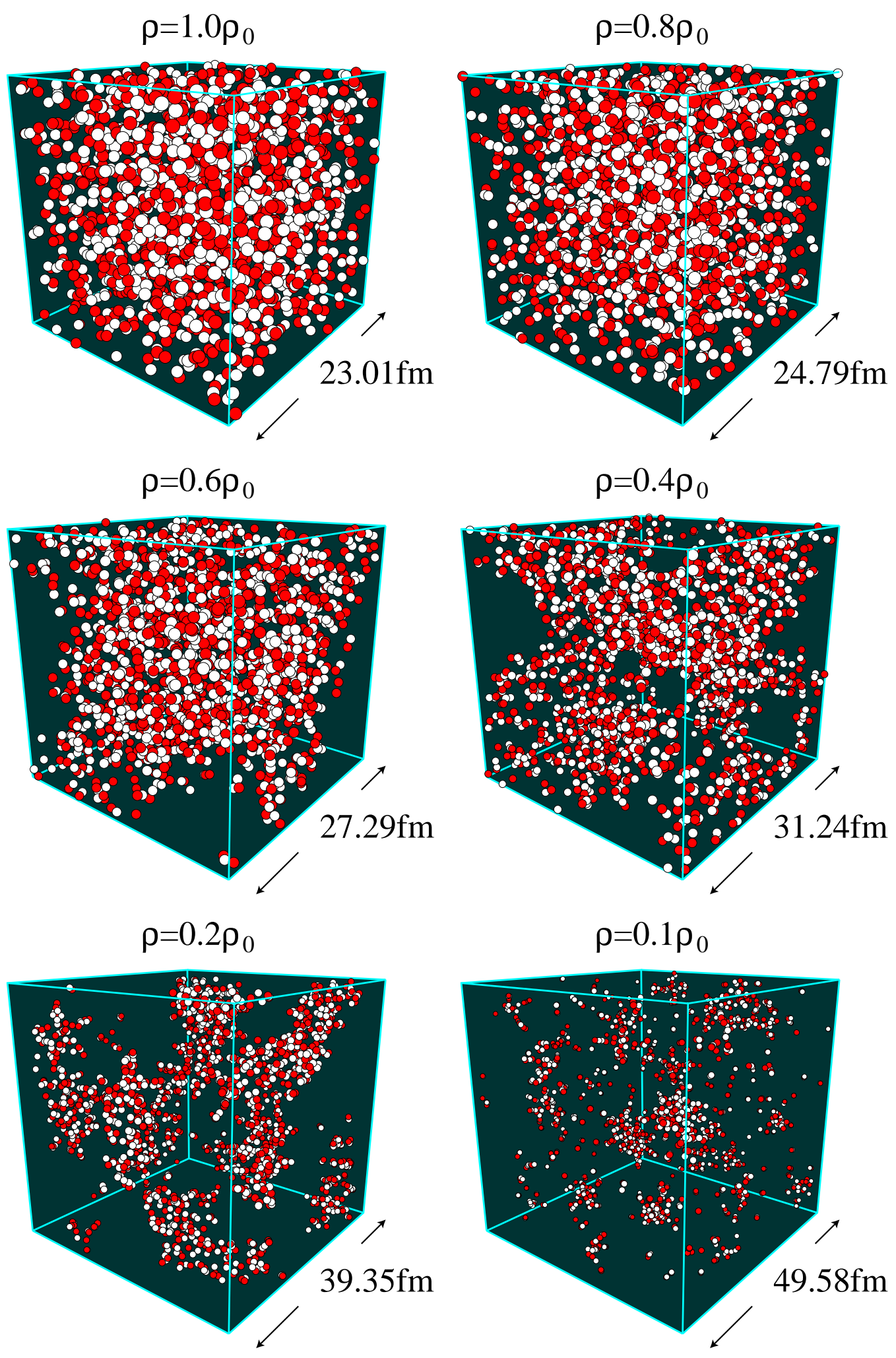

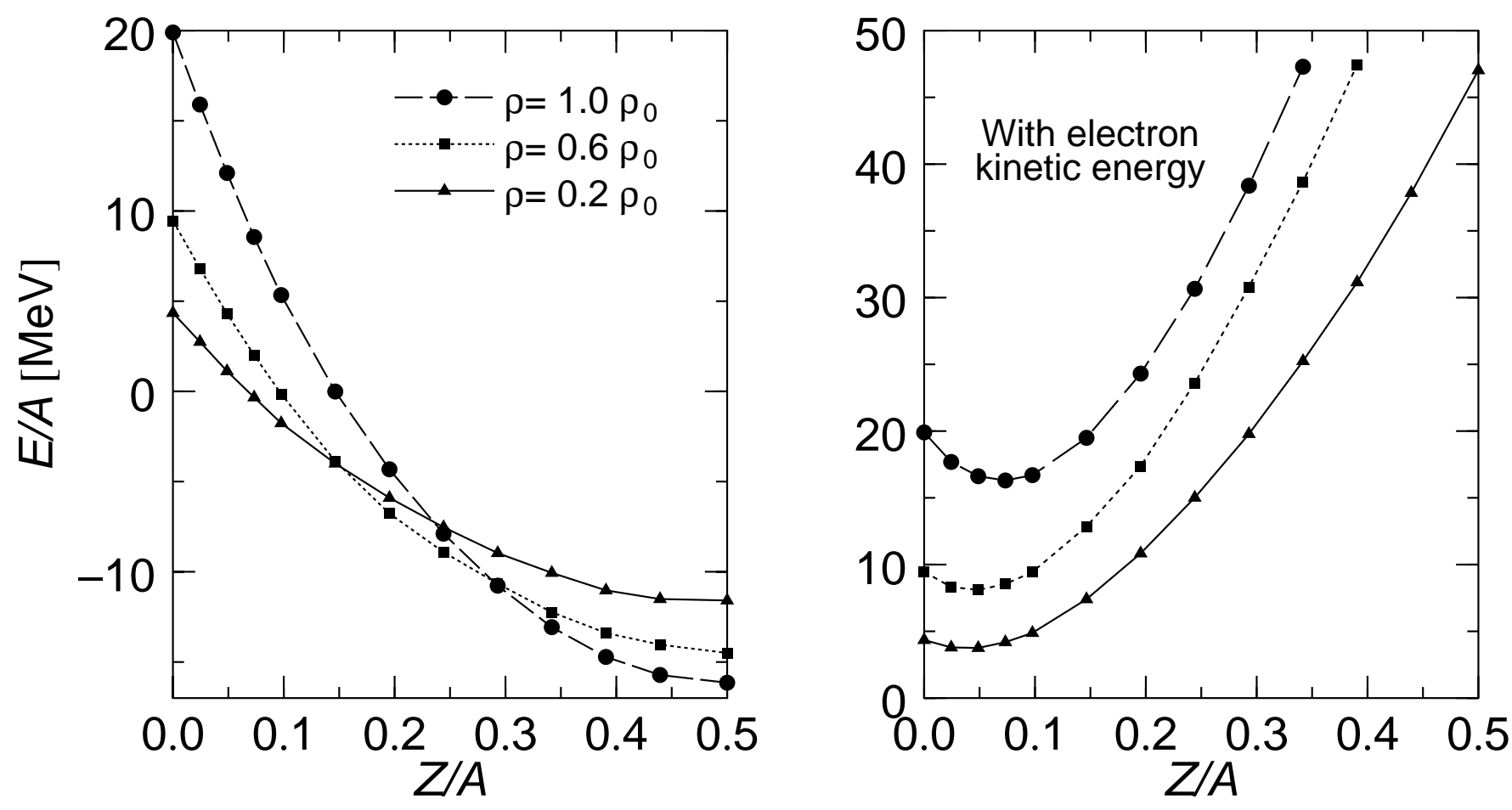
$Z / A=0.098$

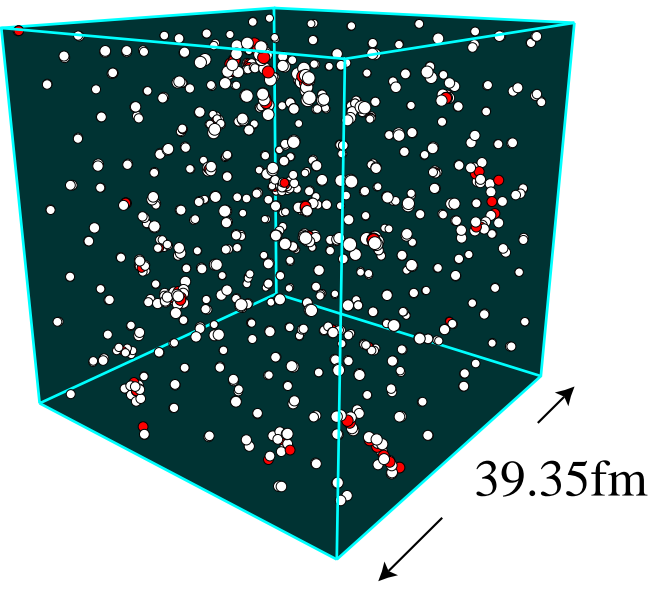

$$
Z / A=0.293
$$

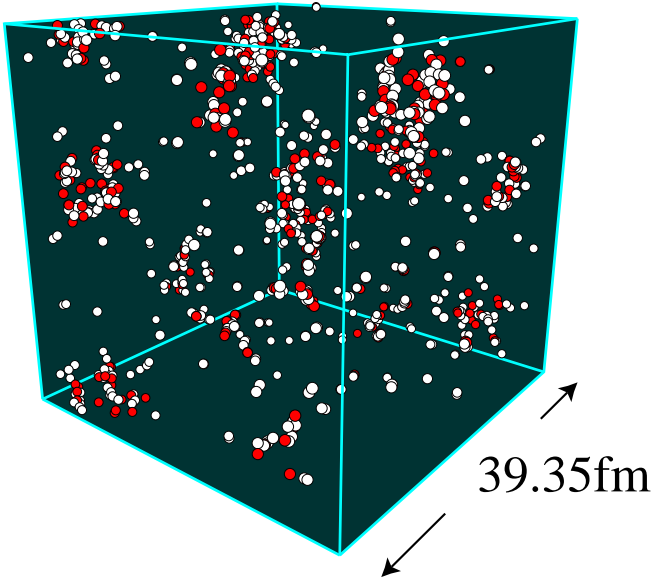

$Z / A=0.195$

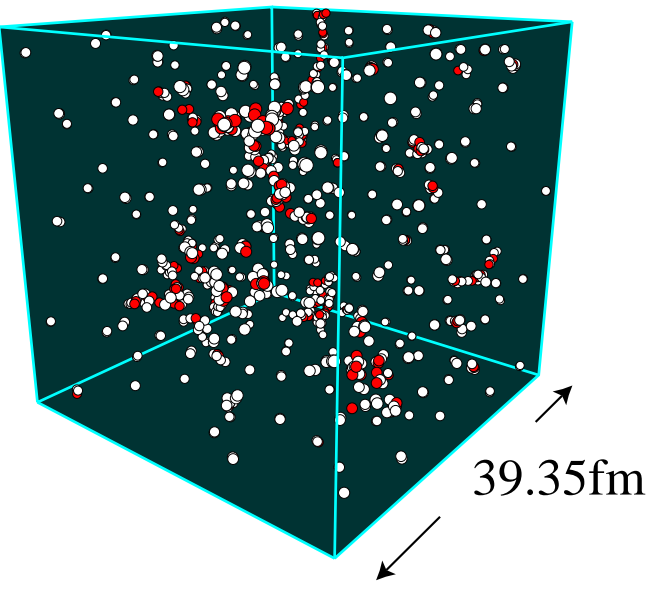

$$
Z / A=0.391
$$

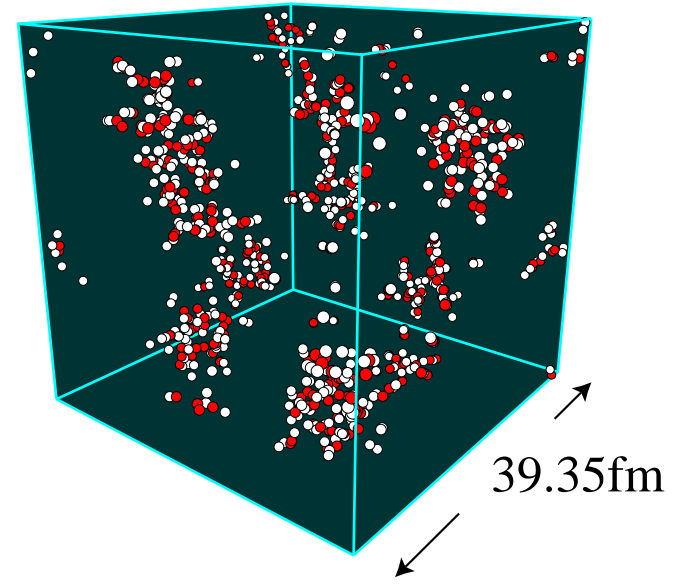

